\title{
Discussion of "A cantilever approach to estimate bending stiffness of buildings affected by tunnelling" by Twana Kamal Haji, Alec M. Marshall, and Walid Tizani
}

\author{
Andrea Franza ${ }^{\mathrm{a}, *}$, Matthew J. DeJong ${ }^{\mathrm{a}}$ \\ ${ }^{a}$ Department of Engineering, University of Cambridge, Trumpington Street, CB2 1PZ \\ Cambridge, United Kingdom.
}

\begin{abstract}
This discussion considers the procedure proposed by Haji, Marshall and Tizani for the assessment of the structural stiffness of frame structures subjected to tunnelling. The discussion focuses on the potential contribution of both shear and bending flexibilities to the response of frame structures to tunnelling, as well as the role of the foundation scheme on the boundary conditions at the base of the structure. The validity of applying the proposed set of equations within currently available methods of prediction of tunnellinginduced deformations, based on modification factors, is also discussed.
\end{abstract}

Keywords: Tunnelling, Soil-Structure Interaction, Building Response

\footnotetext{
*Corresponding author

Email address: andreafranza@gmail.com (Andrea Franza)
} 
In the following, this discussion evaluates [1] the proposed method to estimate the structure stiffness, [2] the assumed displacement boundary conditions for the frame, and [3] the possibility of integrating this method with currently available prediction methods for tunnelling-induced deformations.

[1] To assess the frame stiffness of a linear elastic 3D framed structure subjected to deformations given by a tunnelling-induced settlement trough for an eccentric tunnel-structure configuration the following procedure was implemented at stage 5 . The structure is separated from the soil and foundation. Then, the structure stiffness (i.e. reaction forces induced by nominal displacements) is calculated imposing a mix of force (FBCs) and displacement (DBCs) boundary conditions at the frame base. To replicate the effects of the greenfield settlement trough, vertical FBCs $\left(\mathbf{P}_{\mathbf{z}}\right)$ and fixed vertical DBCs $\left(\mathbf{u}_{\mathbf{z}}=0\right)$ are imposed at the structure base within and outside the tunnel influence zone, respectively, whereas horizontal $\left(\mathbf{u}_{\mathbf{x}}\right)$ and rotational $(\boldsymbol{\Phi})$ DBCs are fixed $\left(\mathbf{u}_{\mathbf{x}}=0, \boldsymbol{\Phi}=0\right)$. This approach is equivalent to defining a condensed stiffness matrix of the superstructure $\left(\mathbf{K}_{\mathbf{c}}\right)$ with respect to the degrees of freedom of the base through FEM analyses. Then, the structure response to tunnelling is characterised by the set of $\mathrm{FBCs} \mathbf{P}^{T}=\left[\begin{array}{llll}\mathbf{P}_{\mathbf{z}} & \mathbf{P}_{\mathbf{x}} & \mathbf{M}\end{array}\right]$ for a given set of DBCs $\mathbf{u}^{T}=\left[\begin{array}{lll}\mathbf{u}_{\mathbf{z}} & \mathbf{u}_{\mathbf{x}} & \boldsymbol{\Phi}\end{array}\right]$ (i.e. $\mathbf{P}=\mathbf{K}_{\mathbf{c}} \mathbf{u}$ ). Subsequently, a scalar value of stiffness $K_{b}$ was obtained by relating $\mathbf{u}_{\mathbf{x}}$ to $\mathbf{P}_{\mathbf{z}}$ as detailed in Equations (6) and (16). This approach allows characterising a given 3D frame with a unique scalar value of stiffness. However, the impact of applying a set of forces $\mathbf{P}_{\mathbf{z}}$ in the region affected by tunnelling rather than a distribution of displacements $\mathbf{u}_{\mathbf{z}}$ equal to the greenfield settlement trough (as previously done by Losacco et al. (2014)) would be of interest.

It is important to clarify that the parameter $K_{b}$, which was defined as the "bending stiffness" by Haji et al. (2018), is a total stiffness derived from the point load analogy given in Eq. (5). As discussed, $K_{b}$ is derived from the condensed stiffness matrix of the structure $\mathbf{K}_{\mathbf{c}}$. In addition, if a Timoshenko beam was used to develop the point load analogy, the total stiffness $K_{b}$ would depend on both the flexural rigidity $E I$ and the ratio between Young's and shear moduli $E / G$, which are related to the bending- and shear-type flexibilities of 3D frame structures. The terms bending- and shear-type flexibilities describes the global deflection response of the frame within a bay as follows: in the bending-type flexibility, the differential settlement between adjacent columns is due to axial deformations of beams/slabs (that relates to the average curvature within a bay); in the shear-type flexibility, this differential settlement is due to deflection of beams/slabs between columns that remain 
vertical. Note that these two terms are not used to indicate the strains of an individual element within the 3D frame (i.e. a single columns or slab span). On the other hand, for the Euler-Bernoulli beam that is adopted to develop the point load analogy (see Equation (4)), the total stiffness is only due to the bending flexibility (i.e. deflection increase is only due to the beam curvature). Although the definition adopted by Haji et al. (2018) is formally correct for the adopted equivalent beam, it may be a source of misunderstanding in the context of geotechnical engineering and tunnelling. Therefore, in this discussion, the parameter $K_{b}$ is referred to as the "total stiffness" to highlight that it does not distinguish between the contributions of shear and bending flexibilities.

In Figure (18), Haji et al. (2018) compared the total stiffness values $K_{b}$ against predictions made through the stiffness assessment method proposed by Franzius et al. (2006). However, the procedure of Franzius et al. (2006) allows estimating a total/equivalent flexural rigidity $E I$ of the structure (that does not account for the shear flexibility), whereas the total stiffness $K_{b}$ also accounts for the shear flexibility. Although the actual structure response to tunnelling depends on the total stiffness, it would be useful to distinguish between these two contributions to define equivalent beams/solids that are meant to represent 3D frames. In point [2], the shape of the structure settlement profile is further discussed.

[2] Haji et al. (2018) does not discuss the physical bases for the assumed DBCs $\left(\mathbf{u}_{\mathbf{x}}=0, \boldsymbol{\Phi}=0\right)$ that, in reality, would be related to the foundation scheme. For raft or continuous strip foundations transverse to the tunnel longitudinal axis, tunnelling-induced differential horizontal movements at the structure base are minimal (Goh and Mair, 2014; Dimmock and Mair, 2008), which is consistent with the DBCs adopted. For separated footing and/or strip foundations orientated along the longitudinal axis, tunnel-structure interaction results in differential horizontal displacements within the foundation (Goh and Mair, 2014; Franza and DeJong, 2017); for these cases, the DBCs analysed by the authors are not representative. Therefore, the horizontal DBCs $\left(\mathbf{u}_{\mathbf{x}}\right)$ considered only apply directly to raft and transverse strip foundations.

On the other hand, the rotational DBCs were also fixed $(\boldsymbol{\Phi}=0)$. Although raft foundation or separated footings may be sufficiently rigid to prevent relative rotations between the column base and the foundation, it is likely that the foundation itself rotate. For long continuous foundations (e.g. rafts or transverse strip foundations), deflections will cause associated rotations 
that vary smoothly with the horizontal offset from the tunnel centreline. For relatively rigid separated foundations, the individual foundations may rotate quite differently from each other, and also quite differently than the local slope of the greenfield settlement profile due to interaction with the structure.

In general, the total structural stiffness at the ground level also depends on the foundation scheme. However, to provide upper and lower bound estimations of the impact of the foundation rotational and horizontal degrees of constraint, further research could assess $K_{b}$ resulting in from four possible combinations of DBCs: $\mathbf{u}_{\mathbf{x}}=$ fixed, released; $\boldsymbol{\Phi}=$ fixed, released.

[3] Previous research reported the variation of the structure deformation shape with respect to the greenfield settlement trough (Farrell et al., 2014; Potts and Addenbrooke, 1997). On the other hand, in the procedure proposed by Haji et al. (2018), the length of structure affected by tunnelling (assumed to behave as a cantilever in Figure (14)) is fixed a priori and does not depend on soil-structure interaction. This assumption could lead to an erroneous estimation of the stiffness. Further research is needed to relate the deformed shape of frames and greenfield input to bending and shear flexibilities.

Although Haji et al. (2018) indicated that the total stiffness value can be used to inform analyses of tunnel-building interaction, it is not fully clear the envisioned application. It is important to consider the applicability of the empirical formulas proposed by Haji et al. (2018) within the modification factor framework (e.g. for computing relative structure-soil stiffness parameters proposed by Franzius et al. (2006) and Giardina et al. (2015), which are needed to estimate deflection ratio modification factors). The design charts for modification factors were developed by modelling equivalent beam/plate structures subjected to tunnelling (which are solids with a lower height-tolength ratio compared to frames with similar EI). These charts are based on the flexural rigidity $E I$ of the equivalent beam/plate rather than a total stiffness value and they do not account for the characteristics of framed structures (Franzius et al., 2006; Giardina et al., 2015; Potts and Addenbrooke, 1997). Also for deep foundations, design envelopes suggested by Franza et al. (2017) relating relative bending stiffness parameter to deflection ratio modification factors do not account for the frame characteristics. Consequently, the proposed empirical relationships could not be safely used within currently available modification factor approaches. Further work is needed to implement the proposed formulas in deformation prediction methods. 
Dimmock, P. S., Mair, R. J., 2008. Effect of building stiffness on tunnellinginduced ground movement. Tunnelling and Underground Space Technology 23 (4), 438-450.

Farrell, R., Mair, R., Sciotti, A., Pigorini, A., 2014. Building response to tunnelling. Soils and Foundations 54 (3), 269-279.

Franza, A., DeJong, M. J., 2017. A simple method to evaluate the response of structures with continuous or separated footings to tunnelling-induced movements. In: Arias, I., Blanco, J. M., Clain, S., Flores, P., Lourenço, P., Ródenas, J. J., Tur, M. (Eds.), Proceeding of the Congress on Numerical Methods in Engineering 2017. Valencia, Spain, pp. 919-931.

Franza, A., Marshall, A. M., Haji, T., Abdelatif, A. O., Carbonari, S., Morici, M., 2017. A simplified elastic analysis of tunnel-piled structure interaction. Tunnelling and Underground Space Technology 61, 104-121.

Franzius, J. N., Potts, D. M., Burland, J. B., 2006. The response of surface structures to tunnel construction. Proceedings of the ICE - Geotechnical Engineering 159 (1), 3-17.

Giardina, G., DeJong, M. J., Mair, R. J., 2015. Interaction between surface structures and tunnelling in sand: Centrifuge and computational modelling. Tunnelling and Underground Space Technology 50, 465-478.

Goh, K. H., Mair, R. J., 2014. Response of framed buildings to excavationinduced movements. Soils and Foundations 54 (3), 250-268.

Haji, T. K., Marshall, A. M., Tizani, W., 2018. A cantilever approach to estimate bending stiffness of buildings affected by tunnelling. Tunnelling and Underground Space Technology 71, 47-61.

Losacco, N., Burghignoli, A., Callisto, L., 2014. Uncoupled evaluation of the structural damage induced by tunnelling. Géotechnique 64 (8), 646-656.

Potts, D. M., Addenbrooke, T. I., 1997. A structure's influence on tunnellinginduced ground movements. Proceedings of the ICE - Geotechnical Engineering 125 (2), 109-125. 